\title{
COMMENTS ON THE DETECTION AND CLASSIFICATION OF COMPACT EMISSION-LINE GALAXIES
}

\author{
T. D. Kinman
}

Kitt Peak National Observatory*

Four methods for finding enission-line galaxies have been compared. Method (a) uses the ultraviolet excess, as found either by filter photography (Haro 1956) or by objective prism spectra (Markarian 1967). Blanco (1974) introduced a thin prism with the CTIO Schmidt (1740 A m-1 at HB) which with IIIa-J plates [Method (b)] gave enough resolution for Smith (1975) and MacAlpine et al. (1977a, $1977 \mathrm{~b})$ to detect and classify galaxies by strong emission lines.

Following a suggestion by McCarthy that even higher dispersion might be useful, I have used the CTIO Schmidt with [Method (c)] the $4^{\circ}$ prism, a GG455 filter and IIIa-J emulsion and with [Method (d)] the $10^{\circ}$ prism, an RG630 filter and IIIa-F emulsion. These latter give about $400 \AA \mathrm{mm}^{-1}$ at $\mathrm{HB}$ and $\mathrm{Ha}$ respectively which improves the visibility of emission lines against the galaxy continuum so that [OIII] 5007 and 4959 and $H \beta$ can be seen on the green plates and $\mathrm{H \alpha}$ and [SII] 6725 can be seen on the red plates.

In a field $\left(13^{\mathrm{h}} 58^{\mathrm{m}},-30^{\circ}\right)$ observed by Smith et al. (1976) methods (c) and (d) recovered all 7 galaxies previous $\overline{1 y}$ found by them and found a dozen more - chiefly by their Ha emission. In the Virgo cluster method (d) discovered 18 new emission-1ine galaxies but failed to recover about half of a group of faint emissionline galaxies which had previously been discovered by their ultraviolet excess. In an equatorial field, studied by MacAlpine et al. (1977b) using method (b), about two-thirds of the quasars found by them are ultraviolet excess objects found by the PHL and other surveys using method (a). MacAlpine et al. found 17 emission-line galaxies in this field while I found $\overline{15}$ using the ultraviolet excess as a criterion; but only six galaxies were in common.

*Operated by the Association of Universities for Research in Astronomy Inc., under contract with the National Science Foundation. 
The ultraviolet excess and the thin-prism technique are useful in finding quasars and high-luminosity galaxies which tend to have low space densities and high redshifts and broad strong lines which may be seen at low dispersion. On the other hand higher dispersions which enhance the visibility of lines such as $\mathrm{H} \alpha$, [OIII] 5007, H $\beta$ and [OII] 3727 against the galaxy continua and allow a more quantitative approach are relevant to finding galaxies with weaker emission lines which are common at low redshifts.

REFERENCES

Blanco, V. M. (1974). Publ. Astr. Soc. Pacific 86, 841.

Haro, G. (1956), Bol. Obs. Tonanzintla y Tacubaya 2, No. 14, 8 . MacAlpine, G. M., Smith, S. B. and Lewis, D. W. (1977a). Astrophys. J. Supplement 34,95 .

MacAlpine, G. M., Lewis, D.W, and Smith, S. B. (1977b). Astrophys. J. Supplement 35, 203.

Markarian, B. E. (1967). Astrofizika 3, 55.

Smith, M. G. (1975). Astrophys. J. $20 \overline{2}, 591$.

Smith, M. G., Aguirre, C. and Zemelman, M. (1976). Astrophys. J. Supplement 32,217 . 


\section{DISCUSSION}

Nandy: Can you give an estimate of the number of emission line galaxies relative to normal galaxies?

Kinman: At the moment $I$ do not have the information to answer this question.

Nandy: I agree with you that the $\mathrm{H} \alpha$ region would be the better spectral region than the ultraviolet for the survey of emission line galaxies, as they are known to have considerable amounts of dust.

Kinman: Yes, particularly for Seyfert galaxies where large Balmer decrements have been found, considerable reddening can be encountered. An example was the bright Seyfert in the $13 \mathrm{~h} 58^{\mathrm{m}},-30^{\circ}$ field which is an edge-on system.

Coyne: How seriously can one attempt to do intrinsic line width measurements on objective prism spectra at these dispersions, since the seeing is varying?

Kinman: Generally one cannot do it at all. The relation which I found between the line width of $\mathrm{H} \alpha$ and the image width on the direct plate of the field shows that only in exceptional cases where the intrinsic line width is of the order of several thous and kilometers per second (as in some Seyfert galaxies), does this intrinsic width make a significant contribution to the observed width.

McCarthy: Could you tell us what your exposure times are, how limited you are in exposure times and what filtering procedure has been followed?

Kinman: An exposure time of 120 minutes was used with a GG455 filter for the IIIa-J plates and a RG630 filter for the IIIa-F plates. Significantly longer exposures would be possible at high galactic latitudes with moonless skies. However, with the $10^{\circ}$ prism in particular, since one is not guiding on-axis, differential field rotation sets a limit on the exposure time, which depends on where one is working in the sky. 